\title{
Afectación funcional y calidad de vida tras cirugía de cáncer rectal
}

\author{
Functional impairment and quality of life after rectal cancer surgery
}

Laura Mora*, Alba Zarate, Xavier Serra-Aracil, Anna Pallisera, Sheila Serra y Salvador Navarro-Soto

Servicio de Cirugía General y del Aparato Digestivo, Hospital Universitari Parc Taulí, Universidad Autónoma de Barcelona, Sabadell (Barcelona), España

\section{Resumen}

Antecedentes: La técnica de referencia de la cirugía rectal sigue siendo la escisión total del mesorrecto (ETM), en la que se aplica la laparoscopia por sus ventajas. El intento de evitar el $17 \%$ de reconversión hace que se apliquen técnicas transanales. La ETM transanal (TaETM) se lleva a cabo por grupos experimentales con buenos resultados oncológicos y de morbimortalidad. Objetivo: Este estudio determina la calidad de vida y la función anorrectal de estos pacientes. Método: Estudio observacional de dos cohortes que compara pacientes intervenidos por tumor rectal mediante TaETM o ETM convencional después de 6 meses mínimo de la reconstrucción del tránsito intestinal. Se aplican los cuestionarios de calidad de vida EORTC-30 y EORTC-29, y el cuestionario de valoración de función anorrectal (LARS score). También se recogen variables generales. Resultados: Entre 2011 y 2014 fueron intervenidos 31 pacientes: 15 en el grupo de ETM y 16 en el de TaETM. No se encuentran diferencias estadísticamente significativas en cuanto a cuestionarios de calidad de vida ni respecto a la función anorrectal. Variables generales estadísticamente significativas: tiempo quirúrgico mayor en el grupo TaETM, e infección nosocomial y fallo de sutura menores en el grupo TaETM. Conclusión: La realización de TaETM obtiene los mismos resultados en cuanto a calidad de vida y función anorrectal que la ETM convencional.

PALABRAS CLAVE: Escisión total del mesorrecto. Cáncer rectal. Funcionalidad. Calidad de vida.

\begin{abstract}
Background: The gold standard of rectal surgery remains total mesorrectal excision (ETM) in which laparoscopy is applied for its advantages. The attempt to avoid $17 \%$ conversion rate implies that transanal techniques are applied. Transanal ETM (TaETM) is performed by experimental groups with good oncological and morbimortality results. Objective: This study determines the quality of life and the anorectal function of these patients. Method: Observational study of two cohorts comparing patients undergoing rectal tumor surgery using TaETM or conventional ETM after a minimum of six months of intestinal transit reconstruction. EORTC-30, EORTC-29 quality of life questionnaires and the anorectal function assessment questionnaire (LARS score) are applied. General variables are also collected. Results: 31 patients between 2011 and 2014: 15 ETM group and 16 TaETM. We do not find statistically significant differences in quality of life questionnaires or in anorectal function. Statistically significant general variables: longer surgical time in the TaETM group. Nosocomial infection and minor suture failure in the TaETM group. Conclusion: The performance of TaETM achieves the same results in terms of quality of life and anorectal function as conventional ETM.
\end{abstract}

KEY WORDS: Total excision of the mesorectum. Rectal cancer. Functionality. Quality of life.

\author{
Correspondencia: \\ *Laura Mora López \\ Unidad de Coloproctología (U. de Coloproctología) \\ Servicio de Cirugía General \\ Hospital Universitario Parc Taulí \\ Parc Taulí, $s / n$ \\ 08208 Sabadell, Barcelona, España \\ E-mail: Lmora@tauli.cat
}

Fecha de recepción: 29-11-2017

Fecha de aceptación: 11-04-2018

DOI://dx.doi.org/10.24875/CIRU.M18000022
Cir Cir. 2018;86:140-147

Contents available at PubMed www.cirugiaycirujanos.com 


\section{Introducción}

En los últimos tiempos ha habido muchos cambios técnicos en el campo de la cirugía del cáncer de recto. A pesar de las innovaciones, el tratamiento de referencia del cáncer rectal sigue siendo la escisión total del mesorrecto (ETM), descrita por Heald ${ }^{1}$ en los años 1980. Entre estos cambios contamos con la laparoscopia, que también se aplica dentro de este campo. Existen estudios que avalan las ventajas de su aplicación en la cirugía rectal, como el estudio COLOR $\|^{2}$. Uno de los problemas relacionados con la aplicación de las técnicas laparoscópicas en la cirugía del cáncer rectal es la tasa de conversión a cirugía abierta: un $17 \%$ según las series. El intento por disminuir esta tasa de conversión y realizar mejoras técnicas en cuanto a la morbilidad posoperatoria y los resultados oncológicos ha llevado a los grupos quirúrgicos a la aplicación de técnicas transanales. Se describen las técnicas de ETM transanal $(\mathrm{TaETM})^{3,4}$, que además aprovecha un orificio natural, el acceso transanal, para la extracción de la pieza de resección. Pueden ser técnicas totalmente transanales (TaETM pura) $)^{5}$ o que aprovechan las ventajas de la laparoscopia en algunos puntos del procedimiento



Actualmente se trata de procedimientos establecidos en grupos experimentados en los que se han demostrado las ventajas posoperatorias a corto plazo y la igualdad en cuanto a resultados oncológicos ${ }^{3,8}$. El objetivo del estudio es determinar la calidad de vida y la función esfinteriana de los pacientes sometidos a esta técnica, y si es igual o mejor que la de los pacientes intervenidos por vía abierta o laparoscópica.

\section{Método}

\section{Tipo de estudio}

Estudio observacional con recogida prospectiva de datos de dos cohortes.

\section{Pacientes}

- Criterios de inclusión: pacientes intervenidos de neoplasia de recto con ausencia de recidiva local 0 a distancia de la enfermedad que fueron sometidos a ETM mediante resección anterior baja (RAB) e ileostomía de protección, a los que se había reconstruido el tránsito intestinal mínimo en los 6 meses previos a la entrada en el estudio. Se dividen en dos grupos: aquellos a los que la RAB se les había realizado por vía abierta o laparoscópica (grupo ETM) y aquellos a los que se les había realizado la RAB vía NOTES (natural orifice transluminal endoscopic surgery) (grupo TaETM).

- Criterios de exclusión: funciones superiores deterioradas, demencia o enfermedad psiquiátrica grave, cirugía paliativa, recidiva local diagnosticada, incapacidad para responder a los cuestionarios.

\section{Variables}

- Generales: se recogieron variables demográficas (edad, sexo, ASA, distancia de la lesión tumoral al margen anal, quimiorradioterapia adyuvante), relacionadas con la cirugía (tiempo quirúrgico, tipo de anastomosis, ostomía de protección), anatomopatológicas (margen distal, margen circunferencial, calidad del mesorrecto, número de adenopatías, estadificación) y posoperatorias (complicaciones según la clasificación de Clavien-Dindo, estancia posoperatoria).

- Estudio de calidad de vida: a todos se les realizó estudio de calidad de vida según los cuestionarios validados al español de la European Organisation for Research and Treatment of Cancer (EORTC-30 y EORTC-29). Se trata de unos cuestionarios diseñados por el grupo de calidad de vida de la EORTC para evaluar la calidad de vida de los pacientes afectos de cáncer. Constan de diferentes escalas de valoración: global, funcional y escalas de síntomas; todas ellas puntúan de 0 a 100. En las escalas de funcionalidad, una mayor puntuación refleja una mejor calidad de vida (excelente: 100-75; buena: 74-50; razonable: 4925; mala: 24-0). En cambio, para las escalas de síntomas, las puntuaciones altas reflejan una elevada intensidad de síntomas (nada: 0-24; un poco: 25-49; bastante: 50-74; mucho: 75-100). Se considera que diferencias de puntuación de 5-10 puntos reflejan diferencias clínicas pequeñas, de 10-20 puntos se trata de diferencias moderadas, y superiores a 20 puntos reflejan diferencias grandes 9,10 . La realización de los cuestionarios fue dirigida por el mismo investigador mediante entrevista directa y fueron aplicados por un grupo de colaboradores de forma ciega sin conocer la técnica quirúrgica realizada en cada caso. 
- Estudio de función esfinteriana: a todos se les realizó el estudio del síndrome de resección anterior también validado al español (LARS score). EI LARS score es un cuestionario diseñado para valorar la disfunción intestinal que sufren los pacientes después de una RAB, basándose en los síntomas y su impacto sobre la calidad de vida. Consiste en cinco ítems relacionados con incontinencia de heces líquidas, frecuencia de movimientos intestinales, fraccionamiento (clustering) de las deposiciones y urgencia defecatoria. Clasifica en tres categorías en función de la intensidad (0-20: no LARS; 21-29: LARS menor; 30-42: LARS mayor) $)^{11,12}$.

\section{Estadística}

La descripción de las variables cuantitativas se hace mediante valores de media y desviación estándar cuando la distribución se considera como normal. En caso contrario, se utilizan valores de mediana, intervalo intercuartílico y rango. Las variables categóricas se describen en números absolutos y porcentajes.

En el análisis estadístico, para las variables cuantitativas utilizamos la prueba $t$ de Student y la $U$ de Mann Whitney cuando no se cumplen los criterios de aplicación de la primera.

En el análisis estadístico de las variables categóricas utilizamos la prueba de ji al cuadrado de Pearson.

Para las escalas de calidad de vida de la EORTC, la comparación de las diferentes dimensiones para cada técnica quirúrgica se ha realizado mediante la prueba $t$ de Student para datos independientes, previo cálculo de la homogeneidad de las varianzas. Para el test de LARS (que usa escala de Likert) se ha usado la prueba de ji al cuadrado, excepto para la escala que valora la repercusión sobre la calidad de vida, en la que dado que se puede asumir que se trata de una variable continua se ha usado la $t$ de Student.

Se ha considerado en todo momento un error alfa de 0.05 y un error beta de 0.2 .

La representación de las escalas de calidad de vida QLQ-C30 y CQC-CR29 se ha realizado mediante gráficos radiales en los que cada dimensión del test se ha situado sobre una línea radial.

Los resultados de las pruebas estadísticas se darán para un valor $\mathrm{p}<0.05$ y con su intervalo de confianza del $95 \%$.
Se han recogido las distintas variables en una base de datos en formato Access y se han analizado sus resultados mediante SPSS 21.0.

El estudio fue aprobado por el Comité de Investigación Clínica del hospital (referencia: 2014672).

\section{Resultados}

Se estudiaron 31 pacientes intervenidos entre 2011 y 2014 que cumplían los criterios de inclusión: 15 en el grupo ETM y 16 en el grupo TaETM (Tabla 1).

En cuanto a las variables generales, no existen diferencias estadísticamente significativas en las variables demográficas ni en las anatomopatológicas. Sí existen diferencias en cuanto al tiempo quirúrgico empleado en la realización de cada técnica, siendo mayor el tiempo requerido para la realización de TaETM de forma estadísticamente significativa. También se obtienen diferencias en las variables relacionadas con las complicaciones posoperatorias: existen diferencias estadísticamente significativas a favor del grupo TaETM en cuanto a complicaciones nosocomiales y a fallo de sutura.

El cuestionario EORTC-C30 nos permite comparar la calidad de vida global entre los pacientes incluidos en nuestro estudio y los valores basales de los pacientes con diagnóstico de cáncer colorrectal que se han obtenido a partir de la EORTC (Fig. 1). En ambos grupos de nuestro estudio se obtuvieron puntuaciones superiores a las basales (74.0 en TaETM y 72.6 en ETM, vs. 60.7 basal). Si comparamos las puntuaciones entre ambos grupos a estudio observamos que la diferencia no resulta estadísticamente significativa ( $p=$ 0.874). Se encontraron diferencias estadísticamente significativas en cuanto a la función social entre ambos grupos a favor del grupo TaETM (89.6 vs. 77.4; $p=$ $0.031)$. Aunque las demás escalas valoradas mediante el cuestionario EORTC-C30 no mostraron diferencias estadísticamente significativas, sí se detectaron diferencias clínicamente relevantes en cuanto a los síntomas de función intestinal. Los pacientes del grupo ETM presentaron mayor puntuación tanto en estreñimiento como en diarrea respecto al grupo TaETM (33.3 vs. 22.9 en estreñimiento y 23.8 vs. 14.6 en diarrea).

$\mathrm{Al}$ analizar los resultados obtenidos mediante el cuestionario EORTC-CR29 no se observaron diferencias estadísticamente significativas ni clínicamente relevantes en ninguna de las escalas funcionales de imagen corporal, ansiedad, peso e interés sexual en hombres ni en mujeres (Fig. 2). En cuanto a las escalas de síntomas, se analizaron aquellos que 
Tabla 1. Características generales de la población estudiada

\begin{tabular}{|c|c|c|c|}
\hline \multirow[t]{2}{*}{ Características } & \multicolumn{2}{|c|}{ Resección anterior baja } & \multirow[t]{2}{*}{$p$} \\
\hline & $\begin{array}{l}\text { Abierta o laparoscópica, } \\
\text { grupo RAB }(n=15)\end{array}$ & $\begin{array}{c}\text { Transanal, } \\
\text { grupo Ta-ETM }(n=16)\end{array}$ & \\
\hline \multicolumn{4}{|l|}{ Demográficas } \\
\hline Edad & 64,00 & 59,94 & 0.439 \\
\hline Sexo & & & 0.704 \\
\hline Hombre & 10 & 12 & \\
\hline Mujer & 5 & 4 & \\
\hline ASA & & & 0.456 \\
\hline । & 0 & 0 & \\
\hline$\|$ & 9 & 11 & \\
\hline III & 6 & 4 & \\
\hline IV & 0 & 1 & \\
\hline Distancia tumor al margen anal & 7,93 & 7,44 & 0.723 \\
\hline Neoadyuvancia & 11 & 7 & 0.247 \\
\hline \multicolumn{4}{|l|}{ Quirúrgicas } \\
\hline Tiempo quirúrgico & 114,29 & 251,88 & 0.000 \\
\hline Tipo anastomosis & & & 0.367 \\
\hline Término-terminal mecánica & 15 & 16 & \\
\hline Ostomía protección (ileostomía) & 15 & 16 & 0 \\
\hline \multicolumn{4}{|l|}{ Anatomopatológicas } \\
\hline Margen distal & 2,16 & 2,43 & 0.579 \\
\hline Margen circunferencial & 1,30 & 15,44 & 0.395 \\
\hline Calidad mesorrecto & & & 0.484 \\
\hline Completo & 14 & 16 & 0 \\
\hline Parcial & 0 & 0 & \\
\hline Incompleto & 1 & 0 & \\
\hline N. ${ }^{\circ}$ adenopatías & $1,07 / 12,79$ & $2,25 / 13,56$ & 0.953 \\
\hline Estadio & & & 0.143 \\
\hline 0 & 3 & 0 & \\
\hline I & 6 & 5 & \\
\hline$\|$ & 3 & 7 & \\
\hline III & 3 & 2 & \\
\hline \multicolumn{4}{|l|}{ Complicaciones posoperatorias } \\
\hline Médicas & $26.7 \%$ & $6.7 \%$ & 0.122 \\
\hline Quirúrgicas & $20 \%$ & $26.7 \%$ & 0.74 \\
\hline Nosocomiales & $60 \%$ & $13.3 \%$ & 0.006 \\
\hline Fallo de sutura & $60 \%$ & $13.3 \%$ & 0.006 \\
\hline Tiempo de estancia hospitalaria (días) & 5.07 & 7.75 & 0.308 \\
\hline
\end{tabular}

también aparecen en el cuestionario de LARS score y solo se observaron diferencias estadísticamente significativas en cuanto a síntomas de dolor y distensión abdominal, siempre superiores en el grupo ETM que en el grupo TaETM. Los pacientes del grupo ETM presentaban mayor frecuencia defecatoria e impotencia, con una diferencia de puntuación clínicamente relevante (superior a 10 puntos) (Fig. 3).

Mediante el cuestionario LARS score se analizó la función anorrectal. El $60 \%$ de los pacientes del grupo ETM presentaron síndrome de resección anterior (LARS) mayor, el $13.3 \%$ presentó LARS menor y el $26.7 \%$ no presentó LARS. Del grupo TaETM, el $62.5 \%$ de los pacientes presentó LARS mayor, el 18.8\% LARS menor y el $18.8 \%$ no presentó LARS. No hubo diferencias estadísticamente significativas en la intensidad del LARS entre ambos grupos (Fig. 4).

\section{Discusión}

Desde hace tiempo se ha demostrado la validez de las técnicas laparoscópicas en el manejo de los tumores rectales, tanto respecto a la morbimortalidad quirúrgica como a los resultados oncológicos, en comparación con los procedimientos clásicos ${ }^{3}$, siempre manteniendo la ETM como el referente de todos los procedimientos con independencia del tipo de abordaje 1 . Con la aparición de las técnicas transanales se ha podido avanzar aún más en la mejora dentro de la aplicación de la laparoscopia en el tratamiento 

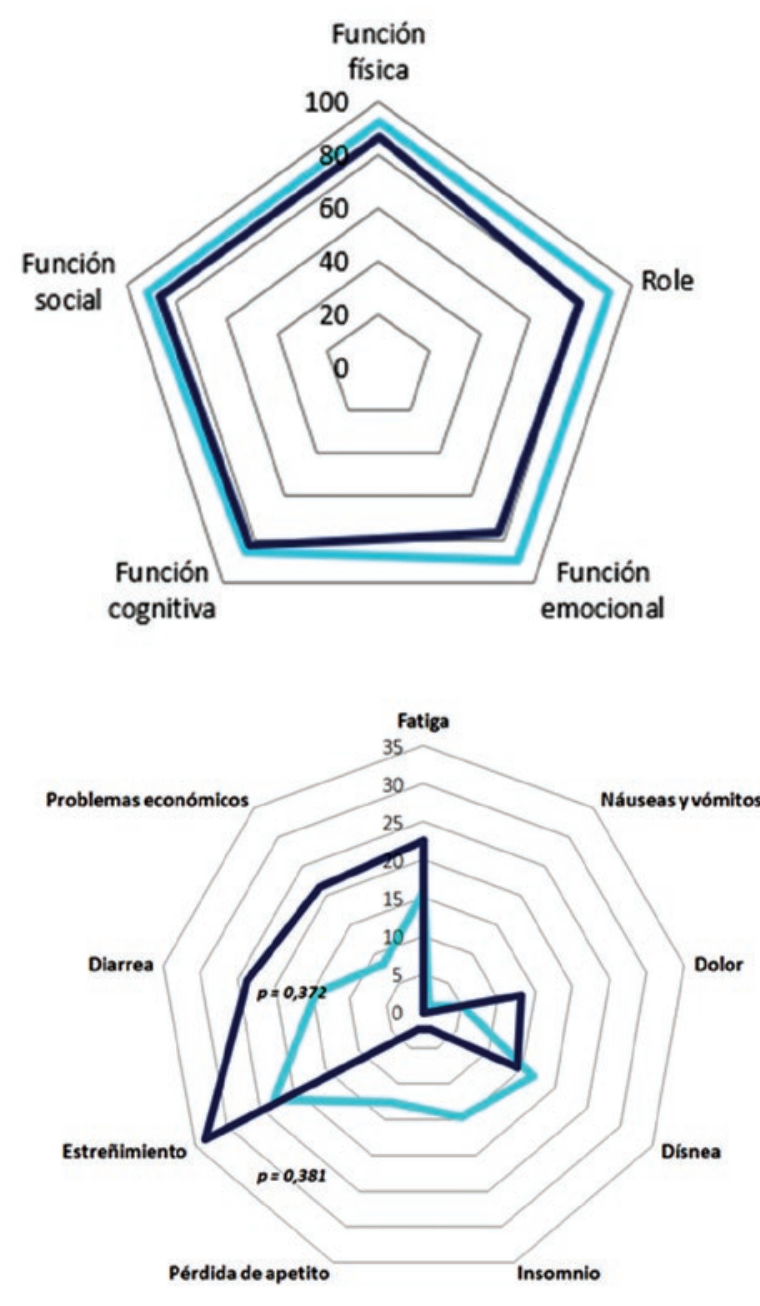

\begin{tabular}{lccc}
\hline & NOTES & RAB & p \\
\hline Escala de calidad de vida & & & \\
Calidad de vida global & 73,96 & 72,62 & 0,874 \\
& & & \\
Escalas funcionales & 92,5 & 86,67 & 0,273 \\
Funcionalidad física & 91,67 & 79,76 & 0,255 \\
Funcionalidad de rol & 89,58 & 77,38 & 0,031 \\
Funcionalidad emocional & 85,42 & 83,33 & 0,775 \\
Cognición & 91,67 & 86,90 & 0,604 \\
Funcionalidad social & & & \\
& & & \\
Escala de síntomas & 15,97 & 22,61 & 0,462 \\
Fatiga & 1,04 & 0 & 0,359 \\
Náuseas y vómitos & 5,20 & 13,09 & 0,235 \\
Dolor & 16,67 & 14,28 & 0,814 \\
Disnea & 14,58 & 21,42 & 0,426 \\
Insomnio & 12,5 & 2,38 & 0,190 \\
Pérdida de apetito & 22,92 & 33,33 & 0,381 \\
Estreñimiento & &
\end{tabular}

Figura 1. Cuestionario EORTC CR-30. Escala global y funcional Análisis estadístico: $t$ de Student.

de los tumores rectales ${ }^{3,4}$. Además de demostrar que técnicamente es posible, también se ha visto que los resultados oncológicos de estas técnicas son equiparables a los estándares marcados clásicamente ${ }^{4}$. Con los resultados de este estudio, aunque con una muestra pequeño, se puede observar un menor número de complicaciones posoperatorias en el grupo TaETM que en el grupo de RAB. Lo que falta por demostrar es si en funcionalidad y calidad de vida la aplicación de estas técnicas iguala o mejora los resultados obtenidos hasta ahora.

Sabemos que la realización de ETM para tratar los tumores rectales lleva asociada una tasa de alteraciones genitourinarias, disfunción sexual y funcionalidad anorrectal nada despreciables. Estudios recientes refieren un $20-30 \%$ de alteraciones genitourinarias y de disfunción sexual en estos pacientes ${ }^{13,14}$. También se describe un $10-30 \%$ de alteraciones en la funcionalidad anorrectal15,16. Todo ello supone alteraciones en la calidad de vida.

La ETM permite unos resultados oncológicos muy buenos en cuanto a supervivencia de los pacientes afectos de cáncer rectal'. También es importante determinar qué procedimientos para realizar la ETM pueden conllevar un empeoramiento o una mejora de la calidad de vida. Creemos que es importante conocer cuáles son los resultados funcionales que ofrecen los avances tecnológicos. Por esta razón hemos llevado a cabo este estudio, para averiguar si los pacientes que se están tratando mediante TaETM presentan variaciones en la calidad de vida en comparación con los pacientes tratados mediante $R A B$ laparoscópica habitual. Se trata de un estudio de cohortes retrospectivo, por lo que no se ha podido realizar aleatorización ni cálculo muestral de los grupos.

Las características generales de los dos grupos estudiados permiten ver que, a pesar de las limitaciones del estudio, estos grupos son parecidos en cuanto a variables demográficas. No hay diferencias respecto a edad media ni sexo, en los dos grupos predominan los pacientes ASA II y III, y en cuanto a los tumores, en los dos grupos la distancia media de la lesión al margen anal es similar, así como la administración de neoadyuvancia. En lo referente a las características quirúrgicas, en los dos grupos todas las anastomosis fueron término-terminales $\mathrm{y}$ a todos los pacientes se les realizó ileostomía de protección. En lo que sí existen diferencias estadísticamente significativas es en el tiempo quirúrgico empleado para cada técnica: la cirugía transanal (grupo TaETM) precisa más tiempo quirúrgico que la cirugía abierta o laparoscópica. Posiblemente estas diferencias puedan ser mejoradas, ya que se trata de la experiencia inicial en cirugía transanal. Las características anatomopatológicas son iguales en 


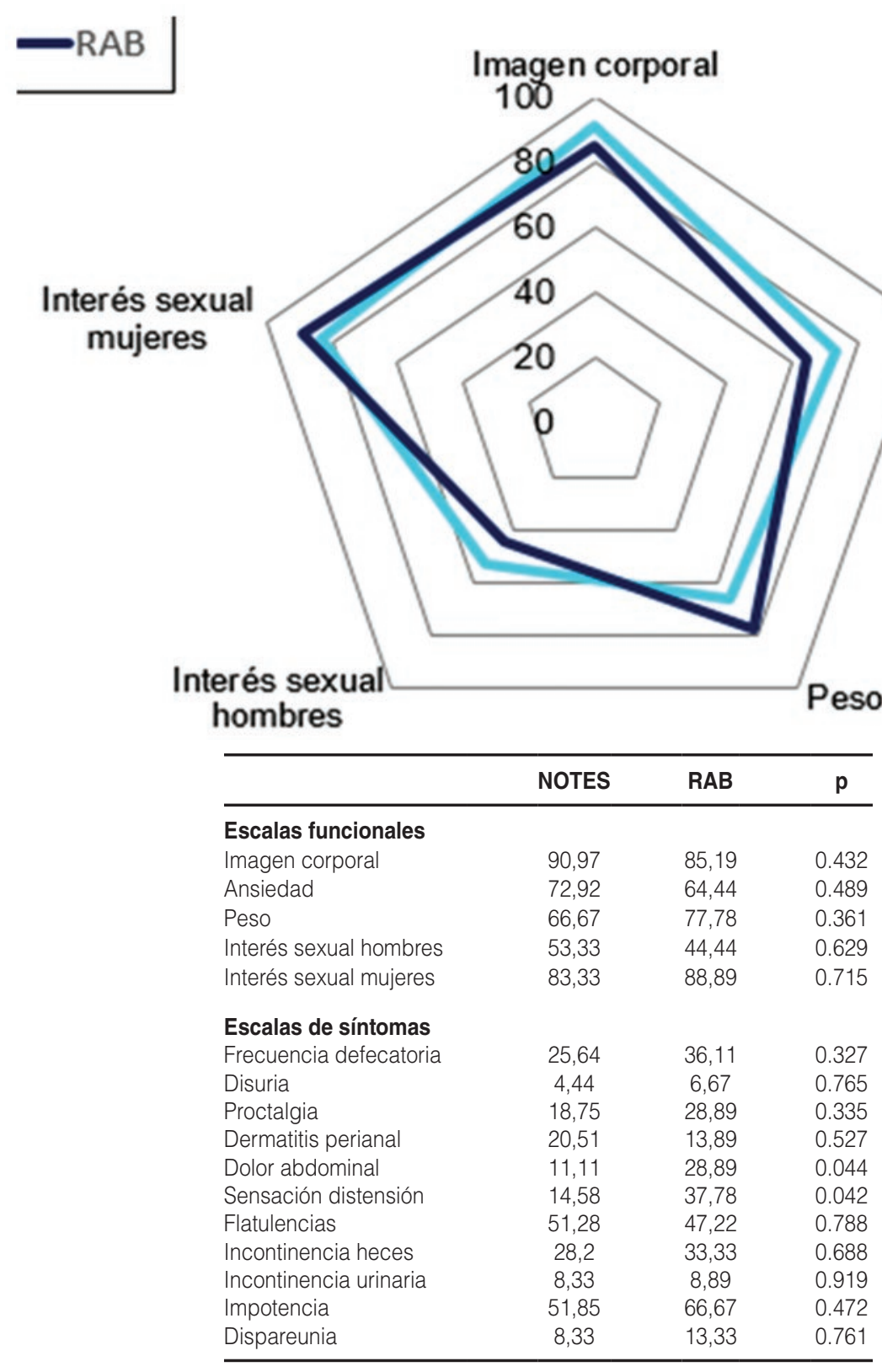

Figura 2. Cuestionario EORTC CR-29. Escalas de función.

los dos grupos. También hay diferencias estadísticamente significativas en las complicaciones posoperatorias. Existen más complicaciones nosocomiales y más fallos de sutura en el grupo de ETM abierta o laparoscópica. Es un número pequeño de pacientes, y tampoco es el motivo de estudio, pero la técnica transanal parece más segura en este aspecto.

En nuestro grupo vemos, según el EORTC-30, que es un cuestionario general sobre la valoración de la calidad de vida, que los dos grupos presentan valores muy parecidos y en ambos casos mejores que los valores de referencia que ofrece la EORTC9. Cuando analizamos el cuestionario específico (EORTC-29) observamos que no hay diferencias entre los dos grupos y que solo hay diferencias clínicas en cuanto a dolor y distensión abdominal, que son peores en el grupo de ETM (dato difícil de interpretar).

En cuanto a la función anorrectal, que valoramos con el cuestionario de LARS, no hay diferencias entre los dos grupos y los valores son comparables a los resultados obtenidos por otros autores ${ }^{17}$. Por tanto, podemos decir que utilizar un rectoscopio para la realización de TaETM no parece tener repercusión sobre la funcionalidad anorrectal, tal como ya demostramos en el grupo de pacientes sometidos a cirugía local de lesiones rectales ${ }^{18}$. 


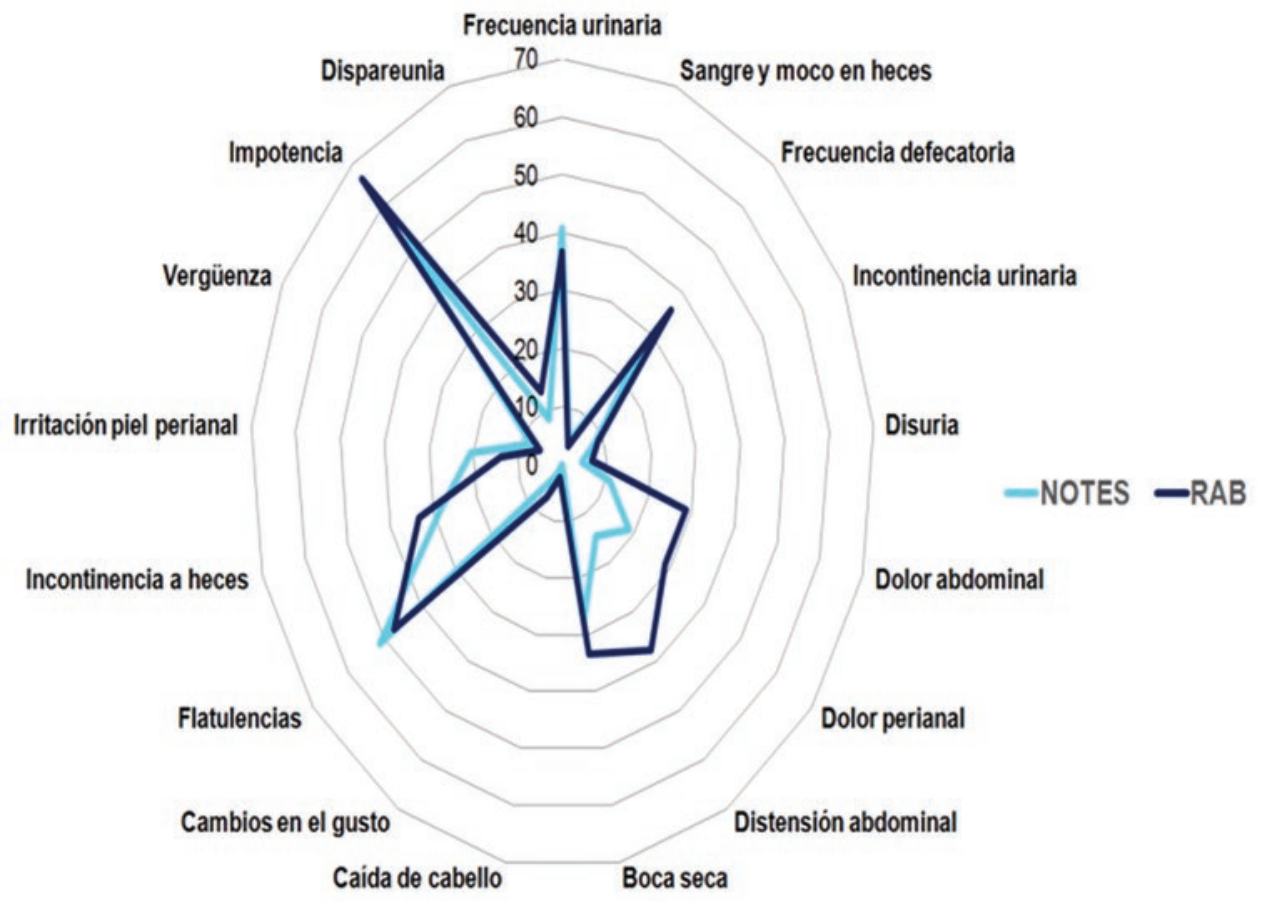

Figura 3. Cuestionario EORTC CR-29. Escala de síntomas

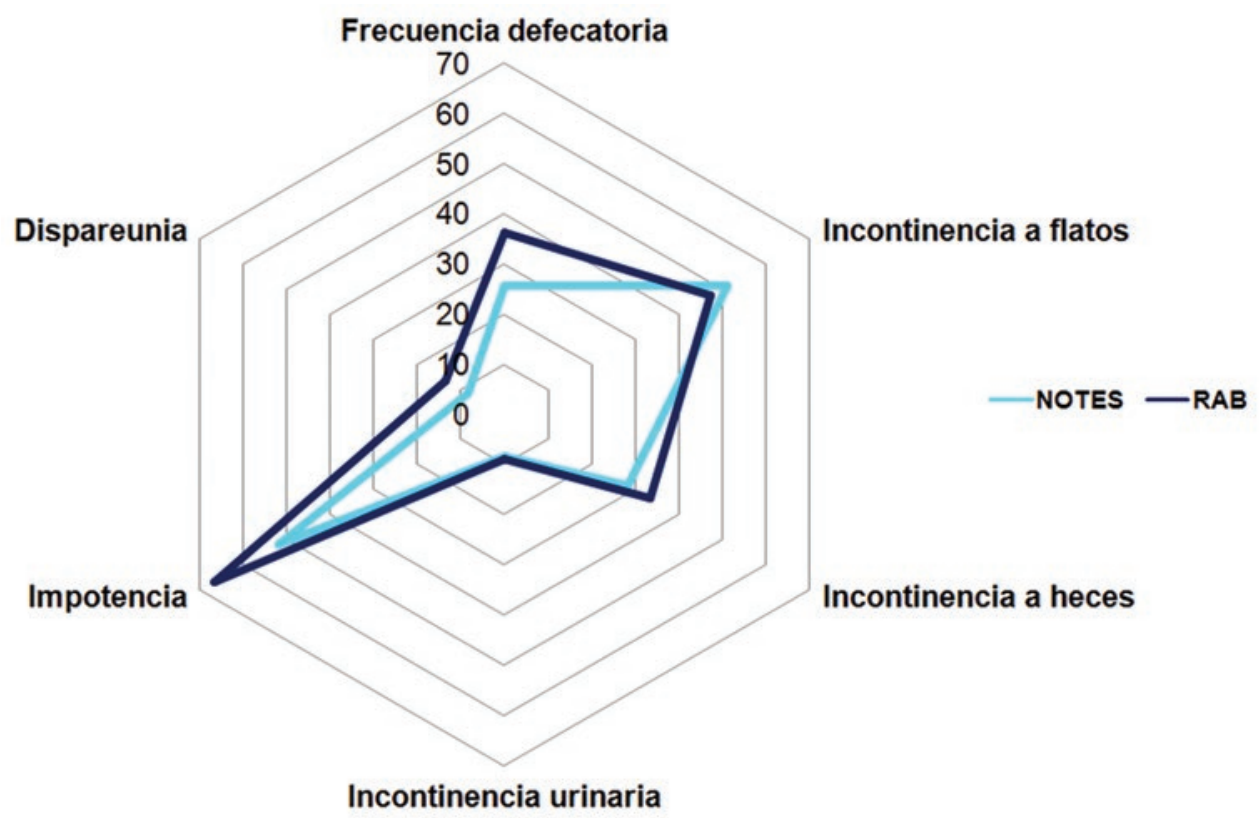

Figura 4. Análisis síntomas EORTC CR-29 que aparecen en el cuestionario LARS.

RAB ( ): RAB transanal, grupo TaETM

NOTES ( $\quad$ ): RAB abierta o laparoscópica, grupo ETM

Análisis estadístico: ji al cuadrado.

Existe poca literatura acerca de la afectación de la calidad de vida y la función anorrectal después de la realización de una TaETM. Se han presentado resultados similares a los nuestros en un grupo de 25 pacientes sometidos a sigmoidectomía transanal, incluso con datos manométricos que muestran que no sufren variaciones en la funcionalidad anorrectal o que estas son transitorias y se recuperan ${ }^{19}$. Existe un estudio en el que se valora la calidad de vida de un grupo de 30 pacientes sometidos a TaETM y les 
aplican los mismos cuestionarios que se utilizan en este trabajo, en dos ocasiones: al mes y a los 6 meses del posoperatorio ${ }^{20}$. Presentan algunas diferencias en cuanto a resultados, con un porcentaje mayor de afectación en el LARS score que nuestro grupo de pacientes a quienes se realiza TaETM. A pesar de las limitaciones que ellos mismos refieren, concluyen que la calidad de vida de los pacientes sometidos a TaETM no es diferente de la de aquellos pacientes a quienes se realiza ETM convencional.

Nuestro estudio presenta limitaciones: tiene pocos pacientes en cada grupo, no existe aleatorización y no se ha podido hacer un cálculo muestral debido a ser un estudio retrospectivo. Son grupos en los que no ha sido posible la homogeneización.

Con los resultados obtenidos, teniendo en cuenta las limitaciones del estudio, podemos decir que hasta el momento los resultados de calidad de vida y de funcionalidad anorrectal de los pacientes sometidos a TaETM no son diferentes a los obtenidos en los pacientes sometidos a ETM convencional. De momento no podemos decir que la técnica produzca un empeoramiento de la calidad de vida o síndrome de resección anterior de los pacientes sometidos a ella. No obstante, serán necesarios estudios prospectivos y aleatorizados que confirmen los resultados obtenidos hasta el momento.

\section{Conflicto de intereses}

Ninguno de los autores presenta ningún tipo de conflicto de intereses en relación con la publicación de este trabajo.

\section{Bibliografía}

1. Heald RJ. The "HolyPlane" of rectal surgery. J R Soc Med. 1988;8:503-8

2. Van der Pas MH, Haglind E, Cuesta MA, Lacy AM, Hop WC, Bonjer HJ, et al. Colorectal cancer Laparoscopic or Open Resection II (COLOR II) Study Group. Laparoscopic versus open surgery for rectal cancer (COLOR II): short-term outcomes of a randomisedphase 3 trial. Lancet Oncol. 2013;14:210-8
3. Sylla P, Rattner DW, Delgado S, Lacy AM. NOTES transanal rectal cancer resection using transanal endoscopic microsurgery and laparoscopic assistance. Surg Endosc. 2010;24:1205-10.

4. Serra-Aracil X, Mora-López L, Casalots A, Pericay C, Guerrero R, Navarro-Soto S. Hybrid NOTES: TEO for transanal total mesorrectal excision: intracorporeal resection and anastomosis. Surg Endosc. 2016;30:346-54

5. Leroy J, Barry BD, Melani A, Mutter D, Marescaux J. No-scar transanal total mesorrectal excision: the last step to pure NOTES for colorectal surgery. JAMA. Surg. 2013;148:226-30.

6. Horgan S, Cullen JP, Talamini MA, Mintz Y, Ferreres A, Jacobsen GR, et al. Natural orifice surgery: initial clinical experience. Surg Endosc. 2009;23:1512-8

7. Lacy AM, Rattner DW, Adelsdorfer C, Tasende MM, Fernández M, Delgado $S$, et al. Transanal natural orifice transluminal endoscopic surgery (NOTES) rectal resection: "down-to-up" total mesorectal excision (TME) - short-term outcomes in the first 20 cases. Surg Endosc. 2013;27:3165-72.

8. Fernández-Hevia M, Delgado $S$, Castells A, Tasende M, Momblan D, Díaz del Gobbo G, et al. Transanal total mesorectal excision in rectal cancer: short-term outcomes in comparison with laparoscopic surgery. Ann Surg. 2015;261:221-7.

9. Arraras Urdaniz JI, Villafranca Iturre E, Arias de la Vega F, Domínguez Domínguez MA, Lainez Milagro N, Manterola Burgaleta A, et al. The EORTC quality of life questionnaire QLQ-C30 (version 3.0). Validation study for Spanish prostate cancer patients. Arch Esp Urol. 2008;61:949-54.

10. Arraras J, Suárez J, Arias de la Vega F, Vera R, Asín G, Arrazubi V, et al. The EORTC Quality of Life questionnaire for patients with colorectal cancer: EORTC QLQ-CR29 validation study for Spanish patients. Clin Transl Oncol. 2011:13:50-6.

11. Emmertsen KJ, Laurberg S. Low anterior resection syndrome score: development and validation of a symptom-based scoring system for bowel dysfunction after low anterior resection for rectal cancer. Ann Surg. 2012;255:922-8

12. Juul T, Ahlberg M, Biondo S, Emmertsen KJ, Espin E, Jiménez LM, et al. International validation of the low anterior resection syndrome score. Ann Surg. 2014;259:728-34.

13. Kneist $\mathrm{W}$, Junginger $\mathrm{T}$. Residual urine volume after total mesorectal excision: an indicator of pelvic autonomic nerve preservation? Results of a case-control study. Colorectal Dis. 2004;6:432-7.

14. Shah EF, Huddy SPJ. A prospective study of genito-urinary dysfunction after surgery for colorectal cancer. Colorectal Dis. 2001;3:122-5.

15. Maas CP, Moriya Y, Steup WH, Klein Kranenbarg E, van de Velde CJ. A prospective study on radical and nerve-preserving surgery for rectal cancer in the Netherlands. Eur J Surg Oncol. 2000;26:751e757.

16. Enker WE, Merchant N, Cohen AM, Lanouette NM, Swalon C, Guillem J, et al. Safety and efficacy of low anterior resection for rectal cancer: 681 consecutive cases from a specialty service. Ann Surg. 1999;230:544-52; discussion 552-4

17. Bregendahl S, Emmertsen KJ, Lous J, Laurberg S. Bowel dysfunction after low anterior resection with and without neoadjuvant therapy for rectal cancer: a population-based cross-sectional study. Colorectal Dis. 2013;15:1130-9.

18. Mora López L, Serra Aracil X, Hermoso Bosch J, Rebasa P, Navarro Soto $S$. Study of anorectal function after transanal endoscopic surgery. Int J Surg. 2015;13:142-7.

19. Steinemann DC, Zerz A, Germann S, Lamm SH. Anorectal function and quality of life after transrectal rigid-hybrid natural orifice transluminal endoscopic sigmoidectomy. J Am Coll Surg. 2016;223:299-307.

20. Koedam TW, van Ramshorst GH, Deijen CL, Elfrink AK, Meijerink WJ, Bonjer $\mathrm{HJ}$, et al. Transanal total mesorectal excision (TaTME) for rectal cancer: effects on patient-reported quality of life and functional outcome. Tech Coloproctol. 2017;21:25-33 